\title{
Research Challenges in Nanosatellite-DTN Networks
}

\author{
Marco Cello, Mario Marchese, and Fabio Patrone \\ University of Genoa, Genoa GE, Italy, \\ marco.cello@unige.it, mario.marchese@unige.it, f.patrone@edu.unige.it, \\ WWW home page: http://Www.scnl.dist.unige.it/
}

\begin{abstract}
Summary. Current approaches based on classical satellite communications, aimed at bringing Internet connectivity to remote and underdeveloped areas, are too expensive and impractical. Nanosatellites architectures with DTN protocol have been proposed as a cost-effective solution to extend the network access in rural and remote areas. In order to guarantee a good service and a large coverage in rural areas, it is necessary to deploy a good number of nanosatellites; consequentially, for reliability and load balancing purposes, is also needed a large number of ground stations (or hot spots) connected on the Internet. During a data connection, a server on the Internet that wants to reply to the user on rural area, has many hot spot alternatives to whom it can deliver data. Different hot spots can send data to final destination with different delivery delay depending on the number, position and buffer occupancy of satellites with which it comes into contact. The problem of choosing the optimal hot spot becomes important because a wrong choice could lead a high delivery delay.
\end{abstract}

Key words: Nanosatellite network, Delay Tolerant Network Architecture, Congestion Control, Next-Hop selection

\section{Introduction}

Despite the worldwide demand of ICT services and the continuous increment of the number of developing countries, currently, only about $40 \%$ of the world population has access to Internet. One of the reasons is that a large amount of people still lives in underdeveloped countries or in remote areas which do not possess ICT infrastructure. The costs needed to connect these areas using cables and common infrastructures are prohibitive compared with the yielded benefits. Satellite communications provide a less expensive way to provide Internet access in these areas. However, current satellite technologies require high costs in the construction, launch and maintenance. Nanosatellites [1] have been recently proposes as a cost-effective solution to extend the network access in rural and remote areas. CubeSat [2], a kind of nanosatellite, is fabricated and launched into low-earth orbit using $0.1 \%$ of the cost of a classical LEO communication satellite. Rural and/or disconnected area will be connected through a local gateway (cold spots) that will communicate in an opportunistic fashion with the nanosatellite 
constellation using the Delay Tolerant Networking (DTN) paradigm. Nanosatellites will carry the data and will send them to the gateways connected to the Internet. On the return path, the central node of the constellation will communicate with servers on the Internet and with the nanosatellite through deployed hot spots that will deliver the data to the rural area.

\section{Related works}

The problem to connect remote areas to the Internet is not a recent challenge. [3] proposes to establish a communication with Internet for the nomadic Saami population who lives in remote areas in Swedish Lapland. The solution uses DTN mobile devices and a series of fixed and mobile relay nodes. In [4] is described DakNet, an ad-hoc wireless network that provides asynchronous connectivity. DakNet is based on rural kiosks to deliver information to users and portable storage devices called Mobile Access Points (MAPs) mounted on a bus, a motorcycle or even a bicycle, which transport data among kiosks and Internet gateways. A similar architecture is described in [5]. The architecture in [6] is a multi-hop mesh network composed of long-distance 802.11 links with high gain directional antennas.

All the described architectures offer valid and inexpensive solutions (e.g. with an investment of $\$ 15$ million, DakNet could equip 50000 rural buses in India), but suffer of severe performance limits and insecurities due to the massive use of ground facilities.

To bypass these drawbacks, satellite networks have been proposed as solution. Iridium [7], Globalstar [8] and Orbcomm [9] are Low Earth Orbit (LEO) satellite constellations that provide satellite phone and low-speed data communications. Inmarsat [10] is a Geostationary Earth Orbit (GEO) satellite constellation that provides voice and data communication services. Nevertheless, these solutions are very expensive due to the production and launch costs. Other solutions involve the use of a network of balloons traveling at an altitude of about $20 \mathrm{kms}$ (Google's Project Loon) [11], and the use of drones in the new Facebook project called Internet.org [12].

A recent solution [1] is represented by the joint use of nanosatellites and DTN paradigm. Nanosatellite is an interesting solution aimed of avoiding the drawbacks of the use of an all-terrestrial network and to reduce the implementation costs of GEO and LEO satellite networks. CubeSat [13] is a nanosatellite: it is a $10 \mathrm{~cm}$ cube with a mass up to $1.33 \mathrm{~kg}$. The main advantage of CubeSats is the reduced cost: the estimated assembly cost per satellite is from $\$ 50000$ to $\$ 100000$, while the estimated launch cost per group of three Cubesats is about $\$ 200000$. The total cost of a possible CubeSat network composed of 150 nanosatellites and 3000 base stations is about $\$ 33$ million with a lifetime of 5 years.

The DTN paradigm, on the other hand, allows supporting end-to-end data exchange between network nodes even when network paths are concatenations of time-disjoint transient communication links. The DTN architecture [14] is based on the introduction of an overlay layer above transport layer protocol which 
allows to handle delays and disruptions at each hop in a path between a sender and a receiver [15]. The principal implementation of DTN is the Bundle Protocol (BP) [16] whose PDU is the bundle.

\section{Motivations and use case scenario}

The access network on rural areas we envision is composed of a constellation of simple, inexpensive nanosatellites that communicate with ground stations through the DTN paradigm. Figure 1 shows a nanosatellites/DTN network scenario: in a rural area, a group of users or nodes $S_{1}, \ldots, S_{N}$ is connected with the node $C S_{1}$. Nodes $C S_{1}$ and $C S_{2}$, referred in the following as cold spot (CS) are located in remote areas and act as Internet gateway for users. They transmit and receive data with nanosatellites $S A T_{1}, S A T_{2}, S A T_{3}$. Node $D$ is the destination node (e.g. a mail server on the Internet). Node $C$ is the control node of the nanosatellite constellation: it contains all the information necessary to manage the network and takes the decisions to improve the performances. Finally, nodes $H S_{1}$ and $H S_{2}$, referred in the following as hot spot (HS), are connected to Internet and able to exchange data with satellites.

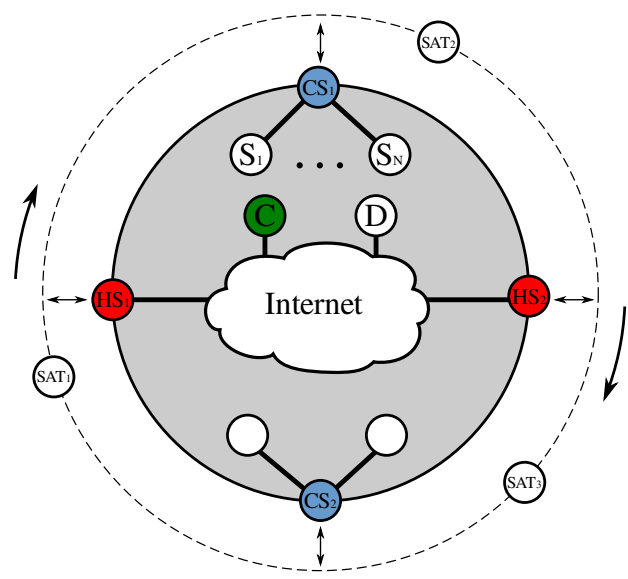

Fig. 1. Nanosatellite network scenario.

Referring to Figure 1, we present a use case scenario in which a user $S_{1}$ located in a rural area wants to access a web page located in a web server $D$ on Internet. User $S_{1}$ sends a DNS request to its default gateway, the cold spot $C S_{1}$, which is also its DNS server. $C S_{1}$ replies to the DNS request pretending to be the web server. $S_{1}$ establishes a TCP connection with $C S_{1}$ and send it the HTTP GET request. $C S_{1}$ replies to $S_{1}$ that the HTTP request has been taken in charge and it will reply as soon as it gets the web page from the web server. $C S_{1}$ encapsulates the HTTP GET message in a bundle destined to central node 
$C$ on Internet and uploads it on the first satellite it comes in contact with (e.g. $S A T_{1}$ ). The bundle is carried by satellite $S A T_{1}$ until it comes in contact with hot spot $H S_{2} . H S_{2}$ receives the bundle and sends it to central node $C$ by using TCP/IP standard protocols. Central node de-encapsulates the bundle to obtain the HTTP GET message and pretending to be the user, starts a TCP connection with the web server $D$. After the reception of the web page, $C$ creates one or more bundles that are forwarded to the selected HS, then to the first satellite that can upload them, and finally delivered to $C S_{1}$. $C S_{1}$ de-encapsulates the bundle and send back the web page to $S_{1}$ by using the same TCP connection of the initial request.

The choice of the hot spot, as said in the introduction, has a direct impact on the delivery time, which should be minimized. This choice can be static (e.g. $C$ always forwards all messages destined to a certain CS to the same HS) or dynamic. Referring to Figure 1, we suppose that 100 bundles are destined to $C S_{1}$ and others 100 bundles are destined to $C S_{2}$. Because of the limited communications performances between hot spot and nanosatellites, only a given amount of data can be uploaded by the HS to the satellite during each contact: in this example we suppose that only 10 bundles can be uploaded. With a static choice, $C$ forwards all 200 bundles to $H S_{1} .10$ satellite contacts are necessary to deliver 100 bundles to $C S_{1}$ and other 10 to deliver 100 bundles to $C S_{2}$ because each satellite in each orbit time can carry only 10 bundles uploaded by $H S_{1}$. Alternatively, with a dynamic selection, $C$ can forward 100 bundles to $H S_{1}$, and 100 to $H S_{2}$ thus doubling the amount of data that each satellite can upload during each orbit. For example, $S A T_{3}$ in one orbit time may receive 10 bundles from $H S_{2}$ and destined to $C S_{2}$ and 10 bundles from $H S_{1}$ and destined to $C S_{1}$.

\section{Research Challenges}

The first challenge is to define a new algorithm whose purpose is to realize a dynamic hot spot selection method in the central node $C$. For each bundle, the central node should compute the optimal hot spot that minimize the delivery time necessary to send the bundle to the destination using information such as the current position of the satellites belonging to the orbit that it manages, and the buffer occupancy of hot spots and satellites.

The second challenge is to realize an architecture (based on [17] and [18]) which ensures a transparent communication between endpoints: users on rural areas make use of standard devices with TCP/IP protocol stack. No no-standard protocols or protocol modifications on the users' devices are allowed. In the same way, server nodes on Internet must use standard protocols. Differently from the literature about DTN, that assume bundle protocol installed on endpoints, for transparent purposes we want to be installed only on cold spots, hot spots, and central node. To do this we need to design a novel architecture able to guarantee on one hand, TCP/IP protocol communications among endpoints and on the 
other hand bundle protocol and satellite-specific transport protocol for the link section. This architecture is illustrated in Figure 2.

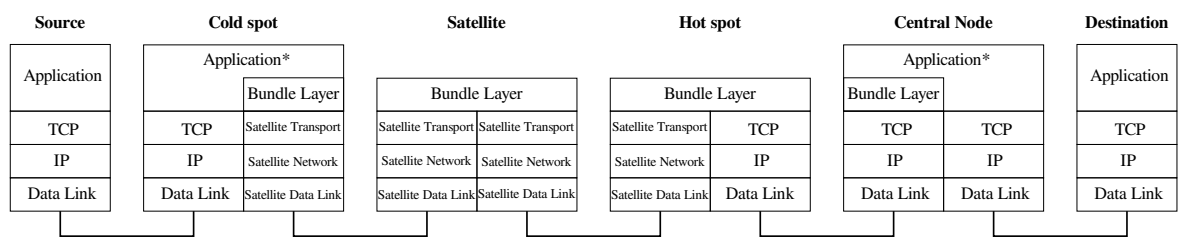

Fig. 2. Network Architecture

\section{References}

1. Burleigh, Scott. "Nanosatellites for universal network access." Proceedings of the 2013 ACM MobiCom workshop on Lowest cost denominator networking for universal access. ACM, 2013.

2. Heidt, H., Puig-Suari, J., Moore, A., Nakasuka, S., and Twiggs, R. CubeSat: A new generation of picosatellite for education and industry low-cost space experimentation. 2000.

3. Doria, Avri, Maria Uden, and D. Pandey. "Providing connectivity to the saami nomadic community." generations 1.2: 3, 2009.

4. Pentland, Alex, Richard Fletcher, and Amir Hasson. "Daknet: Rethinking connectivity in developing nations." Computer 37.1: 78-83, 2004.

5. Seth, A., Kroeker, D., Zaharia, M., Guo, S., and Keshav, S. Low-cost communication for rural internet kiosks using mechanical backhaul. In Proceedings of the 12th annual international conference on Mobile computing and networking: 334345, ACM, 2006.

6. Raman, Bhaskaran, and Kameswari Chebrolu. "Experiences in using WiFi for rural internet in India." Communications Magazine, IEEE 45.1: 104-110, 2007.

7. Iridium Global Network, http://www.iridium.com/About/IridiumGlobalNetwork.aspx

8. Globalstar Network, http://eu.globalstar.com/en/index.php?cid=3300

9. Orbcomm Networks, http://www.orbcomm.com/networks

10. Inmarsat satellites, http://www.inmarsat.com/about-us/our-satellites

11. Google Project Loon, http://www.google.com/loon/

12. Facebook and Partner's Project Internet.org, http://internet.org/

13. Munakata, Riki. "Cubesat design specification rev. 12." The CubeSat Program, California Polytechnic State University 1, 2009.

14. Cerf, V., Burleigh, S., Hooke, A., Torgerson, L., Durst, R., Scott, K., Fall, K., and Weiss, H. Delay-tolerant networking architecture. RFC4838, April 2007.

15. Caini, C., Cruickshank, H., Farrell, S., and Marchese, M. Delay-and disruptiontolerant networking (DTN): an alternative solution for future satellite networking applications. Proceedings of the IEEE, 99(11), 1980-1997, 2011.

16. Burleigh, S., and K. Scott. "Bundle protocol specification." IETF Request for Comments RFC 5050, 2007. 
17. Guo, S., Falaki, M. H., Oliver, E. A., Ur Rahman, S., Seth, A., Zaharia, M. A., and Keshav, S. Very low-cost internet access using KioskNet. ACM SIGCOMM Computer Communication Review, 37(5), 95-100, 2007.

18. Scott, Keith. "Disruption tolerant networking proxies for on-the-move tactical networks." Military Communications Conference, 2005. MILCOM 2005. IEEE. IEEE, 2005. 